\title{
Hidebehind transitando el cuerpo habitado: ejercicios de captura y permanencia
}

\author{
LAURA ROZAS LETELIER
}

Este ensaio analisa a intersecção entre a dança e a historicidade na performance Hidebehind (2018), da coreógrafa brasileira Josefa Pereira, apresentada em 2019 em Lisboa no Teatro do Bairro Alto. Com o seu movimento para trás num movimento circular interrompido pelo gesto, Hidebehind convida-nos a pensar na dança e a interromper o nosso movimento histórico para o questionar activamente. Com base nos princípios da fita de Möbius e da dupla divisão do corpo através do plano coronal, a coreógrafa define na sua operação de captura o ponto de intersecção de várias presenças e temporalidades em simultâneo. O seu corpo, através de mecanismos de repetição e da irrupção do gesto, apresenta-se como um arquivo crítico que desestabiliza e transforma os nossos paradigmas perceptivos, questionando as categorias binárias em vigor no pensamento ocidental. Como transitar o esborramento destes limites na operação de captura? Que potencialidades se encontram na performance para trabalhar com o tempo, recordar e traçar futuros?

CINTA MÖBIUS / CUERPO /ARCHIVO / CAPTURA/ DANZA

No es fácil ser cronopio. Lo sé por razones profundas, por haber tratado de serlo a lo largo de mi vida; conozco los fracasos, las renuncias y las traiciones. Ser fama o esperanza es simple, basta con dejarse ir y la vida hace el resto. Ser cronopio es contrapelo, contraluz, contranovela, contradanza, contratodo, contrabajo, contrafagote, contra y recontra cada día contra cada cosa que los demás aceptany que tiene fuerza de ley.

JULIO CORTÁzAR, Papeles inesperados

Pisada a pisada, desde antes de nuestra llegada habita el espacio una presencia que, apenas vistiendo zapatos, y dividida en un plano dorsal y ventral por medio de una pintura rosa fluorescente, transita de espaldas en insistentes pasadas circulares delineando una órbita bajo la luz nocturna de los focos que desdibujan la supuesta bipartición inscrita en su anatomía. 
Hidebehind $^{1}$ (2018), performance de treinta y cinco minutos, presentada en el marco de la inauguración del Teatro do Bairro Alto durante el 11 al 13 de octubre de 2019 y concluida en el contexto del Programa Avançado de Criação em Artes Performativas (PACAP) es la primera parte de la trilogía "Bestiário PINK". Inspirada en la cinta de Möbius y en la división anatómica del plano coronal, la coreógrafa y performer brasilera Josefa Pereira, residente entre Portugal y Brasil, nos propone mediante su movimiento rebelde un recorrido que imprime en la piel la fuerza del gesto en el encuentro con extraños seres que transitan y habitan su cuerpo. Desde la perspectiva del presente ensayo, Hidebehind nos invita a pensar la danza y a interrumpir nuestro movimiento histórico para practicar una interrogación activa del mismo. Bajo una estética de la repetición y de la duración, el permanente movimiento circular de caminar hacia atrás y contra las manillas del reloj desafía nuestra percepción, abriendo la puerta a cuestionamientos coreo-políticos. Su cuerpo, mediante mecanismos de repetición y en la irrupción del gesto se presenta como un archivo que desestabiliza y transforma nuestros paradigmas perceptivos y cognitivos.

Nos encontramos en una era que inaugura nuevas formas de producción de cuerpos, pensados, bajo una óptica informático-molecular como entes susceptibles de transformación y programación, y en el que el último deseo fáustico supondría la prescindencia del soporte orgánico y material para atravesar sin restricciones tiempos y espacios, lo que ha sido descrito como la emergencia de lo post orgánico (Sibila, 2009). Sin embargo, la materia no puede ser reducida a pura información; el cuerpo entendido en su dimensión sensible se apropia de los recuerdos y los articula como relato estableciendo una dimensión cronotópica y una memoria somática. De modo que la percepción no puede ser vista como una grabación pasiva de la información, sino es inmediatamente unida a potenciales de acción (Violi, 2008: 67).

\section{COREOGRAFIAR LA INTERROGACIÓN HISTÓRICA}

En sulibro Exhaustingdance (2007), André Lepecki, a partir del pensamiento crítico de Peter Sloterdijk respecto a la constitución de la subjetividad

Concepción y performance: Josefa Pereira/ Luz: Aline Santini/ Asistente: Daniel Lühmann/ Duración: $35 \mathrm{~min}$. Pieza concluída en el ámbito de PACAP 1 del Forum Dança (2018). Hidebehind (2018) corresponde a la primera parte de la trilogía en desarrollo Bestiario Pink, seguida por Glimpse (2020) y una tercera parte aún por venir. moderna en su expresión kinética como un continuo "being-towards-movement", reconoce en el desarrollo de la coreografía moderna basada la unión entre danza y movimiento ininterrumpido su correspondencia ${ }^{2}$. Lepecki analiza la traición de esta atadura en las nuevas formas de la danza contemporánea desde la década de los 9o, cuestión que se expresa especialmente en las formas de danza que se han acogido el concepto de "still-acts", entendidos como momentos de suspensión que interrumpen el flujo continuo en la danza y abren la posibilidad a la interrogación histórica. Sin embargo, este stillness, como establece el autor, no implica necesariamente corporeidades estáticas o ausencia de movimiento:

the still-act does not entail rigidity or morbidity. It requires a performance of suspension, a corporeally based interruption of models of imposing flow. The still acts because it interrogates economies of time, because it reveals the possibility of one's agency within controlling regimes of capital, subjectivity, labor, and mobility. (Lepecki, 2007: 15)

Así, si bien Hidebehind no recurre a la inmovilidad como recurso, su movimiento cansa la danza mediante su insistencia y la propuesta de otro "being-towards-movement" afirmando una ética de movilización alternativa, en oposición a la propuesta kinética de la modernidad, de modo que agotar la danza, es agotar el emblema permanente de la modernidad (idem, 2007: 8). Bajo esta óptica, la crisis del movimiento se une a una reflexión acerca de nuestra economía temporal. En este sentido, la danza define su identidad ya no en su pretensión de movimiento fluido, sino en la idea de encuentro, emergiendo nuevas posibilidades en la construcción de las subjetividades.

En relación con lo anterior, Hidebehind mediante la aplicación de los conceptos de plano coronal y cinta Möbius, conjuga una interrelación entre la presencia (supuestamente doble) y el movimiento (de repetir-pasar por el mismo lugar), combinando principios anatómicos y físicos. Por una parte, el plano coronal como división del cuerpo en dos mitades (ventral y dorsal) en función del estudio de las características anatómicas del cuerpo y la determinación de su movimiento, nos presenta un adelante y un atrás, que mediante movimientos de abducción y

2 Por su parte, Carlos Pérez Soto en su libro "Proposiciones en torno a la historia de la danza" (2008, LOM), ha profundizado cómo el capitalismo post-fordista ha condicionado la regulación de los cuerpos, estableciendo conexiones discursivas entre el modernismo expresivista en la danza como funcional al desarrollo capitalista. 
aducción establece los parámetros de su movimiento. Desde una posición biopolítica, se trata de una compartimentación fisiológica que intenta gobernar, entender y controlar el cuerpo humano. Sin embargo, bajo la óptica de la cinta de Möbius, esta supuesta separación dual se vuelve una condición transitoria: en su calidad de "objeto no orientable", derecho y revés en su supuesta oposición se rechazan y absorben como parte de una misma superficie-borde infinitamente continua, a diferencia de un anillo común constituido por dos bordes definidos. Así, el cuerpo de Josefa Pereira condensa dos paradigmas en potencia contradictorios que se encuentran y enfrentan pasando continuamente en marcha atrás. La división del cuerpo se efectúa mediante la aplicación de contraste entre un cuerpo desnudo y a la vez pintado de un llamativo magenta, color que juega con los márgenes de lo visible e invisible al ser un color que no existe en el espectro (Mendoça, 2018). La aplicación de estos paradigmas conlleva consigo implicancias temporales: Hidebehind reúne todos los tiempos, se enfrenta a la catástrofe de espaldas, de modo que las temporalidades atraviesan un cuerpo que transloca frente y reverso, una conjunción de diferentes estados y edades expresados en el juego de los gestos que transcurren la superficie de su cuerpo y extremidades. De modo que:

The past and the future do not designate instants distinct from a supposed present instant, but rather the dimensions of the present itself in so far as it is a contradiction of instants. The present does not have to go outside itself in order to pass from past to future. (Deleuze apud Lepecki, 2016: 129)

En su andar hacia atrás y de espaldas, Josefa Pereira retoma el lugar del "Ángel de la Historia” de Walter Benjamin" (Benjamin, 2012: 173), sin embargo, en su movimiento inverso al transcurso regular del tiempo, esta vez repliega sus alas y decide enfrentarse a la tempestad del progreso y avanzar en contra de la catástrofe a paso firme y decidido. En el camino emergen memorias y ruinas que se encarnan en su piel y se revelan en

3 "Él ha vuelto su rostro hacia el pasado. Donde ante nosotros aparece una cadena de datos, él ve una única catástrofe que amontona incansablemente ruina tras ruina y se las va arrojando a los pies. Bien le gustaría detenerse, despertar a los muertos y recomponer lo destrozado. ángel no puede cerrarlas. Esta tempestad lo empuja incontenible hacia el futuro, al cual vuelve la espalda mientras el cúmulo de ruinas ante él va creciendo hasta el cielo. Lo que llamamos progreso es justamente esta tempestad" (Benjamin, 2012: 173). el gesto. Su cuerpo se presenta así, como un espacio de resistencia que cepilla la historia a contrapelo (idem:172). En este transitar la resistencia no es entendida como reacción, sino como confrontación activa que conlleva una disposición al encuentro con aquellos monstruos.

Hidebehind nos toca por el gesto y contraría nuestros movimientos y geometrías habituales, en este andar para atrás disloca, no solo una dimensión fisiológica, sino también nuestro paradigma cognitivo neuronal $^{4}$. En la medida que la cinta de Möbius como modelo de movimiento desestabiliza nuestras percepciones geométricas, al contrariar la existencia de dos lados y mismo de las concepciones binarias: presencia y ausencia, futuro y pasado, lo ancestral y lo contemporáneo, lo humano y lo animal, entre otras divisiones compartimentadas de nuestra cultura occidental. La incomodidad nos obliga a pensar. Como señala José Antonio Sánchez, la danza contemporánea ha abierto posibilidades para entender la mente como músculo y el pensamiento como algo que circula por el cuerpo (Sánchez: 1999). En este sentido, si bien, su acción no pretende imitación por parte del espectador, sugiere la posibilidad de activar nuestros cuerpos. De este modo, Hidebehind desafía la plasticidad de nuestro cerebro como espectadores: si recorremos la cinta de Möbius necesariamente tendremos que caminar de cabeza abajo.

Hidebehind sugiere la posibilidad de repetición cíclica que, por su parte, produce una densificación y negación del transcurrir del tiempo. La experiencia de la repetición carga consigo dos implicancias o "peligros", por una parte, conlleva la realización de una conciencia reflectiva, es decir, nos vuelve conscientes de nuestra condición de agentes históricos, así como, permite la emergencia de desviaciones (Lepecki, 2016:146). Paso a paso, esta repetición, lejos de domesticar el movimiento, nos sugiere la emergencia de algo ambiguo: "su presentimiento cambia repentinamente en un desarrollo que no se quiere terminar, que resulta imposible de

4 Caminar para atrás, como sabemos, puede producir efectos perjudiciales, sin embargo, su movimiento entrenado podría poseer impactos positivos. El estudio "It takes me back: The mnemonic time-travel effect", realizado por el departamento de psicología de la Universidad de Roehampton investiga los vínculos entre movimiento y pensamiento temporal. En dicho cuáles se les hacía caminar hacia adelante o hacia atrás, para posteriormente efectuárseles pruebas de memoria. Los resultados demostraron, por primera vez, que el viaje en el tiempo pruebas de memoria. Los rast dirigido hacia el pasado inducido por el movimiento mejoró el rendimiento mnemónico mental dirigido hacia el pasado inducido por el movimiento mejoró el rendimiento mnemónico raron, incluso en el caso de que los participantes solo imaginaran el movimiento de caminar hacia atrás, sin realizarlo en la praxis (En Cognition Volume 182, enero 2019, pp. 242-250. En https:// www.sciencedirect.com/science/article/abs/pii/s0010027718302658?via\%3Dihub). 
sintetizar, incontrolado e incontrolable" (Lehmann, 2017: 324, énfasis mío). Esta repetición que podría interpretarse en términos de monotonía y un carácter de refuerzo dramático, es una invitación a mirar más allá, a poner atención en el potencial de las variaciones gestuales, expresadas especialmente en las extremidades superiores de la coreógrafa, brazos y manos amoldan y deforman la carne del rostro y torso. Su cuerpo se transforma en el pasar de plasticidades y resistencias que lo atraviesan. De este modo, la estética de la repetición se presenta como desestructuración y deconstrucción de una fábula (idem, 2017: 324). En el ejercicio de pasar por el mismo lugar emerge la posibilidad creativa de un movimiento errático y la eclosión de la diferencia: "return is the movement that posits a constitutive (creative) difference in repetition" (Deleuze apud Lepecki, 2016: 140).

Es en la fuerza del carácter cíclico del movimiento, que en su demanda física transfiere al espectador la idea de cansancio e incomodidad expresada en esta dualidad truncada de inicio y fin, de pasado y futuro, en que espectadores y performer se sitúan en una situación entre medio, lo que se imprime es un movimiento suspendido en el tiempo. La performance exhibe entonces la posibilidad de un tiempo torcido o sincopado - como señala Rebecca Schneider citando las palabras de Gertrud Stein -, siempre en marcha y anacrónico, en que conviven diferentes economías temporales en simultáneo sobre un cuerpo que es dividido y a la vez indivisible: si realizamos un corte longitudinal en el centro de la cinta de Möbius lo que obtenemos son dos bandas entrelazadas. En este proceso la insistencia lejos de homogenizar se muestra como posibilidad para la irrupción de lo oculto e irregular a la vez que mediante su captura disuelve e imposibilita la articulación de identidades fijas.

Hidebehind é um monstro que habita florestas escuras e que nunca pode ser visto ou ter a sua forma descrita. Por possuir a incrivel habilidade de estar sempre escondido atrás daquele que ousa caminhar por estes bosques ou mesmo atrás dos mais finos troncos de árvores, misteriosamente provoca o desaparecimento para sempre daqueles que consegue capturar. (Cargo Collective website, énfasis mío)
CAPTURA Y TRANSFORMACIÓN: EL VÉRTIGO DEL GESTO

Como un Curupira, criatura protectora de la selva amazónica en la mitología tupí, quien, al tener los pies invertidos desorienta a los cazadores en la persecución de sus huellas (Caracol, 2018), Hidebehind consigue desestabilizar nuestra percepción. De esta forma, "el cuerpo parece desencadenar energías desconocidas u ocultas: se encuentra expuesto, como su propio mensaje y, al mismo tiempo, como algo extremadamente ajeno a sí mismo: [...] Lo siniestro y ajeno al cuerpo es empujado a la superficie (a la piel)" (Lehmann, 2017:354) Por momentos, y ante una luz tenue, la pintura rosa brillante envuelve con su presencia el espacio apoderándose de la figura de la coreógrafa. Mediante esta operación de captura el cuerpo revela el espacio simultáneo de cancelación y aparición [aufhebung]. El pasaje sugiere un carácter ritual, su cuerpo es habitado o bien, poseído por una diversidad de presencias que se encuentran progresivamente en su carne en un proceso dinámico que sugiere el borramiento de los límites de ejercicios de aparición y abandono, destrucción y regeneración. Así, se incriben en su cuerpo transformaciones que circulan en el espacio liminal, "the performance destabilized his perception of himself the others and the reality [...] the crisis induced by the performance can be understood as a crisis of identity as it is experienced in rites of passage" (Fischer-Litche, 2005: 219).

Volviendo al pasaje citado arriba, y considerando la complejidad temporal exhibida en la continua acción de pasar de una entidad que conjuga la simultaneidad de lo binario expuesta por el plano coronal y su supresión o absorción en el modelo de la cinta Möbius, ¿qué implica esta relación de captura por un ser que nunca puede ser visto?

Detrás de la presencia emergen otras sombras, un espacio para lo espectral, que carga con el peso de lo que está solo aparentemente ausente, de lo que se esconde behind. Por una parte, la emergencia de los espectros, pero también el borramiento del cuerpo de la propia coreógrafa. Como propone Rebecca Schneider (2011) en relación al concepto de espectralidad del archivo de Derrida, "in the theatre as in the archive, it is not only the specter that can 'see but not be seen': it is (also and already) the live body bearing the specter across the space, the place, of its consignation" (Schneider, 2011: 110).

La acción de captura supone una separación de lo que es vivido como reducto de lo más propio (mi cuerpo), que es experimentado como ajeno. 
Habitar enquanto força performativa tudo aquilo que ao ser separado é reconhecido como outro. Juntar e reunir tantos outros até que se formem forças anômalas, híbridas, monstruosas capazes de atravessar um corpo que se submete a performá-las, um corpo que se seja tomado/ /possuído. (Cargo Collective website)

El cuerpo se disponibiliza para ser habitado, para el encuentro de otro, sin embargo, esta disponibilidad no es antitética a su resistencia y éste (el cuerpo) no se comporta (o no únicamente) como contenedor definitivo de aquellas presencias. De modo que, no son solo presencias lo que emerge en la composición de la repetición, sino cierta opacidad, "the missed encounter - the reverberations of the overlooked, the missed, the repressed, the seemingly forgotten" (Schneider, 2011: 102). No debemos olvidar, Hidebehind es un monstruo: expresa la inconmensurabilidad, el colapso ante estas presencias provenientes de distintos tiempos en simultaneidad, una producción contra el orden aparentemente regular de la naturaleza o el tiempo linear. Asimismo, como espectro se resiste a su captura estática, su imagen será siempre la de aquello que en su captura arrastra el tiempo y evidencia la borrosidad dada por su continuo pasar.

En este punto, quisiera detenerme en las afirmaciones de los dos pasajes citados, en el doble carácter de un cuerpo que en la misma acción captura y es poseído, que devora y que (transitoriamente) contiene, quizás, contra su voluntad. Si pensamos acción de capturar en sus acepciones de apoderarse de otro y de captar en otro soporte-superficie, podríamos pensar consecutivamente no únicamente en la resistencia y disponibilidad del cuerpo a ser tomado/poseído, sino también en la resistencia que opone lo que es capturado, en la presencia que se resiste a ser capturada, pero que a su vez activamente posee/habita este cuerpo. Esta doble acción podría invitarnos a mirar más allá del binomio presencia/ausencia, así como a mirar el acto de captura -así como de la performance- menos como acto de desaparición, sino como espacio de negociación de esta misma (Schneider, 2011). Tal vez sería mejor interrogarnos sobre qué es lo que aparece en la operación de captura, cómo aparece y qué es lo que se resiste (a aparecer). El cuerpo performea la captura, visto así, la captura no es un acto de desaparición-muerte, sino sistema de transformación-nutrición para mantener(lo) vivo. La captura se revela entonces como el momento de encuentro y el gesto como indicio de este cruce.
Pareciera que dos sistemas en oposición se dan cuerpo aquí, sin querer resolverse: lo que desaparece para siempre, pero que está en constante (re)petición de aparecer. La desaparición para siempre resuena en la amnesia, y se reconoce como la mayor catástrofe. Si bien tal vez la desaparición nunca se pueda inscribir totalmente como tal, si podemos indagar en el acontecimiento de la desaparición y sus mecanismos.

Hidebehind devela la fuerza del gesto y sugiere su ingobernabilidad. Reconocemos en los gestos un sistema culturalmente codificado, sin embargo, su gestualidad difumina nuestros patrones de reconocimiento del gesto, borrándose, en la emergencia de cierta monstruosidad, los límites entre lo humano y lo sobrehumano. Si bien los gestos son intencionados y diseñados para la coreografía, lo que emerge va más allá. Los gestos irrumpen en Hidebehind y detrás de ellos emergen capas de emociones desdibujadas, "esses gestos são como os fósseis em movimento. Têm uma história muito longa e muito inconsciente. Eles sobrevivem em nós" (Didi-Hubermann, 2015: 32). En este caso, en el ejercicio de fusión y borramiento del gesto, lo que surge no son emociones identificables, sino afectos en circulación y entre-medio, "a set of velocities, intensities, and capacities of affecting and being affected" (Lepecki, 2016: 125).

El cuerpo de Josefa Pereira imprime en el gesto micropolíticas del movimiento que develan un cuerpo en constante construcción. La operación del gesto es la operación de la sobrevivencia que se expresa en cada aparición y reaparición que se articula no en su rigidez, sino en su posibilidad de estar en continuo reaparecer a través del movimiento, en el cual el gesto lejos de congelar el tiempo lo actualiza con cada pasada, "still takes place live" (Schneider, 2011: 145). El carácter de los gestos como fósiles en movimiento nos remite a la problemática abordada por Rebecca Schneider acerca del binomio entre lo animado y lo inanimado, entre lo vivo y lo muerto, de la performance como supuestamente dada a desaparecer. En este sentido, aquello que se "esconde siempre detrás de algo y alguien" revela su aparición en el gesto como fósil en marcha en inter-in-animación, de modo que "animate and inanimate, moving and stilled, are not in this sense diametrically opposed as much as part and parcel of an inter(in)animation" (ibidem). 


\section{TRANSITANDO EL ARCHIVO}

El recorrido propuesto conlleva implicancias en nuestra percepción como seres frente a la historia y tensiona las políticas de la recordación y memoria, pero ¿cómo comprender el lugar del cuerpo en el entrecruce entre la interrelación política de la danza y la filosofía de la historia? Tal vez, una aproximación posible a esta pregunta sea entender el cuerpo como archivo, cuestión que, por un lado, amplia las implicaciones de la danza como disciplina artística en su injusta condena al reino de lo efímero y que por otra parte expande las posibilidades del archivo.

La relación entre cuerpo, memoria y archivo ha sido recogida por distintos campos epistemológicos. Desde los estudios de memoria, se propone una memoria inscripta en la materialidad, una lectura bajo la epistemología de la huella, como nos recuerda Paul Ricoeur a través de la metáfora del Theeto de Platón: tal como la impresión en la cera, la memoria amolda la materialidad, inscribiéndose en el cuerpo (Ricoeur apud Contreras, 2012). Por otra parte, desde la semiótica del cuerpo y los afectos la transmisión de la memoria es entendida como huella corporal que deja el contacto con otros cuerpos a modo de un archivo no semántico de la experiencia hacia la sintaxis figurativa ${ }^{5}$ que responde al discurso como "contagio corporal" en que estas huellas no son solo acumulativas, sino también archivables, olvidables, rememorables (Fontanille apud idem). Este carácter plástico de la huella y su potencial de contagio es expresado en Hidebehind por las deformaciones transitorias que la misma coreógrafa efectúa sobre su cuerpo en diferentes ritmos y velocidades.

Sin embargo, la cuestión del cuerpo y el archivo no pasa únicamente por la inscripción de huellas. Lo que es presentado en la performance, revela un completo sistema de transmutación y producción/aparición. En este sentido, sería pertinente la definición de Foucault (1972) del archivo como "sistema de enunciados" que moldean las regularidades específicas de lo decible y la indecible (o de lo visible e invisible), y como un sistema de transformación simultánea de pasado, presente y futuro

5 Desde la semiótica afectiva y encarnada Jacques Fontanille establece en "Soma y Sema" la separación entre carne "Mi" "espacio de resistencia e impulso material, asi como posición de referende la identidad del cuerpo-actante. El Si (cuerpo propio) sería, entre otras cosas, la memoria del Mi (la carne). La sintaxis figurativa es entendida así, como la manera en que el actante adquiere forma mediante la interacción de un sistema material y las fuerzas y presiones que se ejercen sobre él. Dilatación y comprensión van creando estabilidad e inestabilidad. La presencia se revela relacional y tensiva, en cuanto se construye mediante afectos (Fontanille, 2016). que permite recrear la economía de lo temporal en su conjunto (Foucault apud Lepecki, 2013). En este punto, el cuerpo de la coreógrafa no exhibe lo visible, sino la continua negociación entre lo visible y lo invisible. Por otra parte, la falta o desaparición, como critica Lepecki en relación con otros autores como Hal Foster, no debe entenderse como una obsesión por capturar el pasado, por rescatar el tiempo en una relación melancólica con este mismo.

Mediante la resistencia del movimiento y su dimensión afectiva, la propuesta de Hidebehind nos aleja de una visión nostálgica de la danza como "vanishing point" (Lepecki, 2013: 125), de la tradición sentada en la efimeridad de la danza como acción que en el momento de su enunciación se desvanece. En este sentido, en la performance presentada la duración viene dada no tanto por el tiempo del trabajo, un poco más de media hora, sino por su potencial repetición infinita y su insistencia en la acción de pasar - pisada a pisada: pasando, (re)pasando - que amplia el sentido del presente, el que se expresa como coexistencia y no simplemente como una sucesión de "ahoras" (Lepecki, 2007: 131). Esta coexistencia se reafirma en un recorrido que se propone afectivo e íntimo, Josefa Pereira traza un recorrido en solitario, una reflexión en cifrada en lo personal, pero que es compartido y devela un universal. La coreógrafa navega en sus trazos personales, a la vez que encarna diferentes encuentros de manera transitoria que nos transporta a significaciones que sobrepasan su propio cuerpo.

En su estudio sobre el reenactment, Performance Remains (2011), Rebecca Schneider, siguiendo a Derrida propone una relectura del entendimiento del archivo como "house-arrested"-domiciliado y de la lógica patriarcal del archivo. Schneider propone una inversión de esta lógica, en que el problema no sería lo efímero del performance, sino la limitación del entendimiento del archivo, que favorece a aquellos fenómenos que decantan en objetos. De este modo el archivo es visto como una forma de performance de acceso a la historia. En este sentido, Hidebehind expone la captura como momento problemático de intersección del acto continuo de "house-arrest" que performea y hace aparecer la desaparición y su resistencia. La performance se convierte en el espacio de negociación de la ausencia que "provoca el desaparecimiento de aquellos que consigue capturar", pero esa desaparición no es contraria al permanecer, sino que termina por desafiar esa pérdida (Schneider, 2011: 102). Así, pasos en reversa, el cuerpo se convierte en un agente de la historia que trabaja en y a través del tiempo. 
Pues si algo sabe la coreografía, es que un archivo no almacena: actúa. Y sus acciones tienen lugar ante todo mediante la delimitación de zonas de temporalidad y ritmos de presencia, tal como debe hacer la coreografía: "El análisis del archivo comporta, pues, una región privilegiada: a la vez próxima a nosotros, pero diferente de nuestra actualidad, es la orla del tiempo que rodea nuestro presente, que se cierne sobre él y que lo indica en su alteridad; es lo que, fuera de nosotros, nos delimita". (Foucault apud Lepecki, 2013)

Hidebehind reuniendo las figuras del plano coronal y la cinta Möbius, con su único borde, nos invita a reflexionar sobre el carácter del archivo como espacio fronterizo. La cinta Möbius desafía el estatuto de la frontera y nos arroja al encuentro perpetuo. Son los espectadores se sitúan en círculo, delimitando las fronteras de su movimiento y el propio borde de la performance que se mueve en los márgenes impulsada por una fuerza centrífuga en que el cuerpo se desplaza casi rozando a los asistentes, como si una fuerza ajena la expulsara del centro hacia la periferia. Con cada vuelta la performance escribe y re-escribe sus contornos, definiendo una zona de contacto, una superficie para el encuentro entre distintas singularidades y afectos. De modo que la performance se inscribe como un acontecimiento que "pasa alrededor", a través y entre los diferentes cuerpos (Lepecki, 2016: 129). Cabe preguntarse, ¿no son también los propios espectadores quiénes son capturados en este transitar?

Finalmente, si hemos de considerar el cuerpo desde una lógica archivística, sería en el sentido de un archivo siempre en desvío. La teórica Anna María Guasch, desde el campo de las artes visuales, ha diferenciado dos máquinas de archivo: unos son los archivos que siguen un orden delimitado por ciertas leyes y un orden topográfico y otros son los archivos que "acentúan los procesos derivados de las acciones contradictorias de almacenar y guardar, y, a la vez, de olvidar y destruir las huellas del pasado, una manera discontinua y en ocasiones pulsional que actúa según un principio anómico (sin ley)" (Guasch, 2011: 15). Así, el movimiento de Josefa Pereira podría moverse por las lógicas de esta segunda máquina de archivo, en su pasaje emergen y desaparecen los afectos transitorios e ingobernables que atraviesan el cuerpo.

Último paso atrás, la coreografía llega a su fin. El tiempo suspendido se pone en marcha adelante nuevamente, sin embargo, nosotros ya no somos exactamente los mismos. Hidebehind despierta nuestros músculos adormecidos y nos recuerda las múltiples posibilidades de movimientos y subjetividades alternas que pueden transitar nuestro cuerpo, vislumbrando su potencial como espacio de subversión de nuestros paradigmas personales, así como colectivos. Su trayecto rebelde y radical no interroga sobre posibles nuevas políticas de la memoria y la recordación, compromiso que requiere por nuestra parte aventurarnos a caminar por los bosques oscuros e ir al encuentro de los monstruos. Finalmente, no importa si caminar en círculos nos conduce o no a un fin, lo que nos queda son los trazos de un proceso de transmutación y descubrimiento que no pretendemos dominar y que abre la puerta a futuros inciertos. Un círculo que no se termina de cerrar sobre sí mismo y que seguirá volviendo en nuestros cuerpos.

\section{REFERÊNCIAS BIBLIOGRÁFICAS}

BENJAMIN, Walter (2012), Escritos Políticos, Madrid, Abada Editores.

CARACOL, Bruno (2018), Cumplicidades em Arte Capital, https://www.artecapital.net/perspetiva-207-bruno-caracol-cumplicidades, 18 de abril de 2018.

CONTRERAS, María José (2012), "Introducción a la semiótica del cuerpo: Presencia, enunciación encarnada y memoria", Cátedra de Artes $\mathrm{N}^{0_{12}}$, Facultad de Artes, Pontificia Universidad Católica de Chile, pp. 13-29. DIDI-HUBERMANN, Georges (2015), Que emocãa! Que emocão?, Lisboa, KKYM.

FISCHER-LICнтE, Erika (2005), Theater, Sacrifice, Ritual. Exploringforms ofpolitical theater, New York, Routledge. FONTANILLE, Jacques (2016), Soma y Sema: Figuras Semióticas del cuerpo, edición digital, septiembre de 2016, Universidad de Lima, Fondo Editorial.

GUASCH, Ana María (2011), Arte y Archivo, Madrid, Ediciones Akal.

Lehmann, Hans-Thies (2017), Teatro Pós-Dramático, Lisboa, Orfeu Negro.

LEPECKI, André (2016), Singularities: Dance in the Age of Performance, New York, Routledge.

- (2013), "El cuerpo como archivo: El deseo de recreación y las supervivencias de las danzas, in En Lecturas sobre danza y coreografía, Madrid, ARTEA Editoral.

- (2007), Exhausting Dance. Performance and the politics of movement, Wiltshire, The Cromwell Press.

MENDOÇA, Bruno (2018), «Sobre a estreia na peça no SESC Belezinho, São Paulo (setembro 2018)», in programa Teatro do Bairro Alto, octubre 2019.

SÁNCHEZ, José Antonio (ed.) (1999), «Desviaciones» (documentación de la 2a Edición de Desviaciones), Madrid-Cuenca, pp. 13-28.

SCHNEIDER, Rebecca (2011), Performing Remains, art and war in times of the theatrical reenactment, New York, Routledge.

VIOLI, Patrizia (2008), Beyond the body: towards a full embodied semiosis, en Body, language and mind, Berlin, Walter de Gruyter. 


\section{LAURA ROZAS LETELIER}

Membro do CIMa/e (Centro de Investigación y Memoria de las artes escénicas, Chile), tem trabalhado na área da educação em Direitos Humanos e como investigadora em temas relacionados com memória, corpo e arquivo nas artes performativas. Frequenta actualmente o mestrado em Estudos de Teatro na Universidade de Lisboa. 